\title{
Exercise and internet-based cognitive-behavioural therapy for depression: multicentre randomised controlled trial with 12-month follow-up
}

Mats Hallgren, Björg Helgadóttir, Matthew P. Herring, Zangin Zeebari, Nils Lindefors, Viktor Kaldo, Agneta Öjehagen and Yvonne Forsell

\section{Background}

Evidence-based treatment of depression continues to grow, but successful treatment and maintenance of treatment response remains limited.

\section{Aims}

To compare the effectiveness of exercise, internet-based cognitive-behavioural therapy (ICBT) and usual care for depression.

\section{Method}

A multicentre, three-group parallel, randomised controlled trial was conducted with assessment at 3 months (posttreatment) and 12 months (primary end-point). Outcome assessors were masked to group allocation. Computergenerated allocation was performed externally in blocks of 36 and the ratio of participants per group was 1:1:1. In total, 945 adults with mild to moderate depression aged 18-71 years were recruited from primary healthcare centres located throughout Sweden. Participants were randomly assigned to one of three 12-week interventions: supervised group exercise, clinician-supported ICBT or usual care by a physician. The primary outcome was depression severity assessed by the Montgomery-Åsberg Depression Rating Scale (MADRS).

\section{Results}

The response rate at 12-month follow-up was $84 \%$. Depression severity reduced significantly in all three treatment groups in a quadratic trend over time. Mean differences in MADRS score at 12 months were 12.1 (ICBT), 11.4 (exercise) and 9.7 (usual care). At the primary end-point the group $\times$ time interaction was significant for both exercise and ICBT. Effect sizes for both interventions were small to moderate.

\section{Conclusions}

The long-term treatment effects reported here suggest that prescribed exercise and clinician-supported ICBT should be considered for the treatment of mild to moderate depression in adults.

\section{Declaration of interest}

None.

\section{Copyright and usage}

(c) The Royal College of Psychiatrists 2016.
There are compelling reasons to investigate alternative treatment options for depression. Most depressed individuals never seek professional care; among those who do, only half appear to benefit. ${ }^{1}$ Adherence to medication regimens is often poor, ${ }^{2}$ and cognitive-behavioural therapy (CBT) is not always available or affordable. Treatment delays are common and can lead to more entrenched symptoms and a worse long-term prognosis. Additional treatment options are needed that are easily prescribed, readily available and have few negative side-effects. To date, few studies have examined the effectiveness of alternative treatments for depression in large, community-based randomised controlled trials (RCTs). Importantly, treatment effects measured beyond the intervention period are particularly lacking. These long-term outcomes are highly relevant indicators of both effectiveness and the maintenance of treatment response. Exercise is a promising stand-alone treatment for mild to moderate depression. Recent reviews have concluded that exercise is effective in reducing depressive symptoms among people who are depressed or chronically ill, ${ }^{3,4}$ and in otherwise healthy individuals. ${ }^{5}$ Posttreatment effect sizes are comparable to those of psychological and pharmaceutical treatments. ${ }^{3}$ Plausible mechanisms for these benefits include increased serotonin synthesis, hippocampal cell proliferation, ${ }^{6,7}$ reduced levels of pro-inflammatory cytokines, ${ }^{8}$ distraction, mastery and improved self-efficacy. ${ }^{9}$

Internet-based treatments for depression are expanding, ${ }^{10}$ and offer the advantage of immediate accessibility. CBT is a first-line empirically supported treatment for depression, ${ }^{11}$ which can be administered over the internet (ICBT) as guided self-help with limited online support from a therapist. Recent studies suggest that ICBT can be as effective in treating mild to moderate depression as face-to-face $\mathrm{CBT},{ }^{12}$ and that tailored web-based interventions may confer additional treatment benefits in patients with high comorbidity. ${ }^{13}$ Although exercise and ICBT represent promising alternative treatments for depression, evidence regarding their comparative and long-term effectiveness remains scarce. Recent reviews highlight the need for well-powered effectiveness trials that include long-term assessments of treatment response. ${ }^{3,14}$ To overcome this research gap, and with the aim of providing updated, clinically relevant information, we quantified and compared the long-term effectiveness of exercise and clinician-supported ICBT with usual care for depression. The study population consisted of Swedish adults with mild to moderate depression, and the primary end-point was the 12month follow-up assessment. The study was registered with the German Clinical Trials Register (DRKS00008745). The trial protocol can be accessed by searching 'Protokol' via the website www.regassa.se/.

\section{Method}

A multicentre, single-blind, three-group parallel RCT was used to examine the long-term effectiveness of three 12-week interventions for mild to moderate depression: exercise, clinician-supported 
ICBT and usual treatment by a physician in primary healthcare. Assessments were conducted at baseline (pre-randomisation), 3 months later (interim assessment) and at 12-month follow-up (end-point). A priori, we proposed to use intention-to-treat (ITT) analysis. The ethical review board at the Karolinska Institute approved the study (Dnr 2010/1779-31/4). All patients provided written informed consent. A description of the method and interim findings has been published previously. ${ }^{15}$

\section{Patient recruitment}

Participants were recruited through primary healthcare facilities in six county councils in Sweden (Stockholm, Skåne, Västra Götaland, Kronoberg, Blekinge and Västmanland). The selection of regions helped to ensure that the sample included participants from locations varying in population size and composition. Patients aged 18 years or over who scored more than 9 on the Patient Health Questionnaire (PHQ-9) were invited to participate. ${ }^{16}$ Recruitment began in February 2011 and the last participants finished treatment in March 2013. People with a severe somatic illness, primary alcohol or drug use disorder, or who required specialist psychiatric treatment (e.g. for psychosis), were excluded from the trial. Potentially eligible patients were initially referred by their primary healthcare provider, following which trained research assistants (qualified nurses) formally assessed trial eligibility by conducting a standardised diagnostic interview and administered the baseline questionnaires. The principal investigator (a consultant psychiatrist) trained approximately 30 research assistants - all qualified nurses - before the study began to ensure that patients were interviewed consistently. Another member of the research team (a psychiatric nurse) trained the research assistants in the whole examination procedure, including information given to the participants, obtaining informed consent and filling in the questionnaires. Generally this training took 1-2 days. The consultant psychiatrist and psychiatric nurse were available to answer any questions the research assistants had about the process, and during the initial stages of the trial they were contacted regularly.

\section{Randomisation and masking}

Following the baseline assessment participants were randomised to one of three 12-week parallel treatments in blocks of 36 to achieve an even group distribution. The randomisation procedure was performed externally by an independent organisation, the Karolinska Trial Alliance, and individual patients were treated as the 'unit' of randomisation. A computer generated the random group allocation, making the ratio of participants per treatment group 1:1:1. Participants were specifically advised not to discuss their treatment with the assessors, who were kept unaware of group allocation. Information on patients who declined to participate was unknown; it was not feasible for the primary care centres involved in the trial to collect these data. In total, 945 patients met the inclusion criteria, agreed to participate and were included in the baseline analyses.

\section{Follow-up assessments}

Patients were invited to attend a 3-month (post-treatment) and 12-month (end-point) follow-up interview. Interviews were conducted by research assistants at the patient's healthcare clinic or by telephone. Participants who did not return a follow-up questionnaire were contacted by telephone at least twice. As the focus of this trial was on long-term treatment effects, concerted efforts were made to optimise the 12-month response rate with telephone calls and text messages sent to participants after working hours.

\section{Measures}

\section{Primary outcome}

Depression severity was assessed using the clinician-rated Montgomery-Åsberg Depression Rating Scale (MADRS). ${ }^{17}$ All the research assistants strictly followed the instructions given by the authors of this scale; interrater reliability was checked and confirmed to be high. Ten symptoms were rated: reported sadness, apparent sadness, inner tension, reduced sleep, reduced appetite, concentration difficulties, lassitude, inability to feel, pessimistic thoughts and suicidal thoughts. Total scores range between 0 and 60 . Snaith et al proposed a severity categorisation based on the following ranges: $35-60$ severe, $20-34$ moderate, 7-19 mild and $0-6$ remission. ${ }^{18}$

\section{Screening}

The PHQ-9 assessed the presence of depression during the past 2 weeks. ${ }^{16}$ The complete Mini-International Neuropsychiatric Interview (MINI) assessed psychiatric disorders, based on the DSM-IV. ${ }^{19}$

\section{Baseline, interim and end-point assessments}

The EuroQol EQ-5D assessed patients' overall health status, including their capacity to undertake 'usual activities', 'pain/ discomfort' and 'worry/sadness. ${ }^{20}$ Employment status was determined by a single question (nine response alternatives). The Alcohol Use Disorders Identification Test (AUDIT) assessed hazardous drinking $(8+$ men, $6+$ women) and binge drinking (six or more standard drinks consumed on a single occasion, with a frequency of once a month or more) based on recommended cut-off levels. ${ }^{21,22}$ Tobacco use was assessed by asking: 'Do you smoke or use tobacco daily?' Psychotropic and other medications (past 2 weeks) were recorded at baseline.

\section{Interventions}

\section{Exercise}

Patients in the exercise group were further randomised to one of three supervised exercise conditions: light exercise (yoga/ stretching classes), moderate exercise (an intermediate aerobics class) and vigorous exercise (a higher-intensity aerobics/ bodyweight strength training class). All exercise sessions were delivered by qualified trainers with a certificate in personal training. All supervised classes were completed at Friskis och Svettis, a modern fitness centre with locations throughout Sweden (www.friskissvettis.se). In accordance with recommended physical activity levels, ${ }^{23}$ patients were requested to complete three 60 min sessions per week for 12 weeks. Sessions typically included 5-20 participants. Adherence to the exercise regimen was monitored through weekly meetings with a trainer or physiotherapist. Attendance was also checked electronically: entry to individual exercise sessions was recorded in a database, and patients could only access the exercise facility by swiping a membership card at the entrance. The participants also provided data from pulse watches. Participants who did not attend any scheduled sessions for 1 week or more were contacted by telephone and encouraged to resume treatment. Patients completed on average 12 out of the recommended 36 sessions (adherence rate 33\%). 


\section{Internet-based CBT}

Patients randomised to ICBT received treatment through a secure website operated through the county council. The programme was created by qualified, accredited clinical psychologists (5-year tertiary education in Sweden) with 1.5 years of CBT education and training. The content was built partly on the ICBT package described by Johansson et $a l,{ }^{13}$ but was edited and rearranged substantially. Treatment involved the patient working through a self-help manual available online in the form of modules. The manual was mostly text-based, but also included images and sound clips. Before commencing the treatment, all participants in this group received a short telephone call from their assigned clinician (a psychologist) who explained the process. Initially, several online forms were completed to identify patient-specific mental health concerns and work-related problems. The modules completed during the first few weeks addressed problems related to depressive symptoms in general, such as inactivity and avoidance behaviours. Subsequent modules were patient-specific and targeted different comorbid symptoms often seen in depression, such as worry, panic attacks, social anxiety, stress, insomnia and pain. Other patient-specific modules focused on problems related to the workplace and motivated the patient to return to work or find a new job. Throughout treatment, patient responses were monitored by the assigned clinician on a weekly basis so that high-risk individuals could receive additional help if necessary. Cooperation with therapy was monitored electronically, and on a weekly basis by a psychologist. Patients could only move on to the next module if all parts of the previous module had been completed, and all log-ins were recorded. Participants who were inactive for at least a week were contacted by their assigned psychologist and encouraged to continue. In total, patients sent 18 messages to their assigned therapist via a secure online messaging system, received 17 messages and accessed 7.8 (s.d. = 5) online modules out of an expected 13 (adherence rate $60 \%$ ). Additional information about the role of online therapists is presented in online supplement DS1.

\section{Treatment as usual}

Participants randomised to usual care received standard treatment for depression administered by their primary care physican. In most instances, 'usual care' consisted of 45-60 min CBT delivered by an accredited psychologist or counsellor. During the trial patients made on average $8.2($ s.d. $=6.4)$ visits to their general medical practitioner. In total, $22 \%$ reported visiting a psychiatrist, psychologist or psychotherapist (mean 3.3 times) and 10\% reported visiting a counsellor (mean 2.9 times) outside primary care. Patients reported various other health-related treatments during the trial, including visits to a specialist medical practitioner (17\%), physiotherapist (17\%), chiropractor (14\%), healer $(9 \%)$ and self-help group or other treatment (8\%). In total $27 \%$ of patients in this group reported receiving no formal psychological treatment.

\section{Statistical analysis}

An a priori power analysis showed that an estimated sample size of 310 participants per condition would provide approximately $90 \%$ power (two-tailed $\alpha=0.05$ ) to detect a small but meaningful effect size of -0.3 , based on the approximate mid-point of standardised mean differences reported by Cooney et al in a recent Cochrane review of exercise for depression, ${ }^{3}$ and an anticipated withdrawal rate of $25 \%$ recently reported by Pfaff et al. ${ }^{24}$ Data were cross-checked for accuracy and any discrepancies resolved. Missing internal data on key variables were minimal $(<1 \%)$ with one exception: $72 \%$ of responses to MADRS item 2 were missing at 12 -month follow-up (similarly, $23 \%$ from 3 months). This occurred because this item is an observational rating of 'apparent sadness' and $72 \%$ of the 12 -month interviews were conducted by telephone. Apart from this item there was no pattern associated with the missing data. As the first two items of the MADRS were highly correlated $(r=0.72)$ we imputed missing values for item 2 with the response given on item 1 . Where two or more items were missing (including item 2) the MADRS score was dropped. A sensitivity analysis was also performed to compare the imputed data with complete case data by carrying forward the last observation.

Differences in baseline characteristics were assessed using independent samples $t$-tests for continuous data and chi-squared tests for categorical data. ITT analysis was applied. Thus, all participant data were analysed according to the original allocation, regardless of deviations from the assigned treatment or noncooperation. To adjust for baseline group differences in depression severity $(P=0.036)$, MADRS scores were standardised using the mean and standard deviation from baseline within each treatment group. Two approaches were used to examine between-group differences for the change in depression severity. First, mixed effects linear regression models with maximum likelihood estimation were applied to examine the change in standardised depression scores as a function of randomisation group, time and group $\times$ time interaction. A positive quadratic effect of time was observed where scores reduced more from baseline to 3 months compared with the change from 3 months to 12 months $(P<0.0001)$, indicating a convergence in scores over time. Residuals were normally distributed. The square of the correlation between predicted and original standardised scores was 0.32 . This method was also applied in a subgroup analysis restricted to patients with concurrent depression and anxiety (that is, excluding people with a diagnosis of depression only or anxiety only). Second, using a more conservative method, a binary outcome variable was created and mixed effects logistic regression was applied to examine the proportion of participants with 3-month and 12-month depression severity scores more than 1 s.d. below the baseline group average. Both transformation procedures (standardisation and 1 s.d. reduction) brought the three treatment groups to a comparable baseline level. In all analyses we also adjusted for baseline group differences in antidepressant use $(P=0.064)$, age and gender. Parameter estimates are presented for the linear model (step 1) and odds ratios (OR) for the logistic model (step 2). Last, we examined treatment group differences in the proportion of participants reducing their initial MADRS score by $50 \%$ or more at 12 months.

To quantify the magnitude of change in depression severity, Hedges' $g$ effect sizes were calculated by subtracting the mean change from baseline for the usual care group from the mean change from baseline for exercise or ICBT and dividing the difference by the pooled baseline standard deviation. ${ }^{25}$ Effect sizes were calculated such that a reduction in depression severity resulted in a positive effect size. All analyses were performed using SPSS version 22 .

\section{Results}

Of the 945 patients, $740(78 \%)$ returned a 3-month questionnaire and $797(84 \%)$ returned a 12-month questionnaire (Fig. 1). The proportions of those not responding were similar: usual care $n=56(18 \%)$, exercise $n=49(15 \%)$ and ICBT $n=42(13 \%)$. There was no difference based on gender, depression severity or employment status at baseline. Study drop-out was mainly 


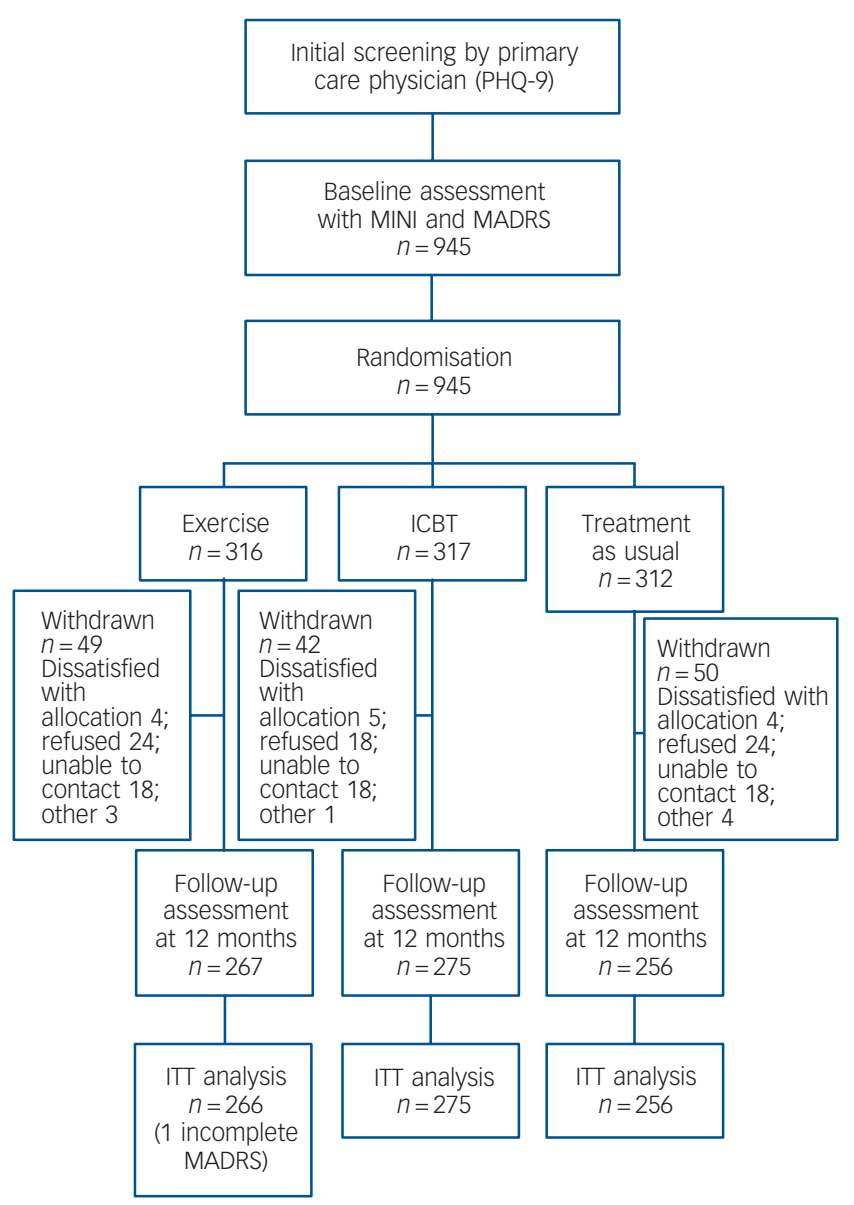

Fig. 1 Participants' progress through the trial. ICBT, internetbased cognitive-behavioural therapy; ITT, intention to treat; MADRS, Montgomery-Åsberg Depression Rating Scale; MINI, Mini-International Neuropsychiatric Interview; PHQ-9, Patient Health Questionnaire.

attributable to not being able to reach participants despite repeated attempts. A smaller proportion withdrew from the study because they were dissatisfied with the randomisation outcome. Participant characteristics are shown in Table 1. Raw scores on the primary outcome (depression severity) are shown in Table 2. Most participants were women $(73 \%)$; the sample's mean age was 43 years $($ s.d. $=12)$ and most had a concurrent depressive and anxiety disorder. Many patients also reported somatic complaints, physical pain and difficulties with daily living. Twenty-seven per cent of participants were taking antidepressants during the trial. The proportion within the exercise and ICBT groups $(30 \%)$ slightly exceeded that in the usual care group (24\%; $P=0.064)$. The use of other medications (e.g. contraceptives) during the trial was minimal.

\begin{tabular}{|c|c|c|c|}
\hline Characteristic & $\begin{array}{l}\text { Physical } \\
\text { exercise }\end{array}$ & ICBT & $\begin{array}{l}\text { Treatment } \\
\text { as usual }\end{array}$ \\
\hline Age, years: mean (s.d.) & $43(12)$ & $43(12)$ & $43(12)$ \\
\hline \multicolumn{4}{|l|}{ Gender, $n(\%)$} \\
\hline Female & $220(70)$ & $229(72)$ & $240(77)$ \\
\hline Male & $96(30)$ & $88(28)$ & $72(23)$ \\
\hline \multicolumn{4}{|l|}{ Country of birth, $n(\%)$} \\
\hline Sweden & $249(79)$ & $253(80)$ & $249(81)$ \\
\hline Elsewhere & $67(21)$ & $63(20)$ & 60 (19) \\
\hline \multicolumn{4}{|l|}{ MINI diagnosis, $n(\%)$} \\
\hline Depressive episode & $225(73)$ & $224(72)$ & $217(70)$ \\
\hline Recurrent depressive disorder & $189(61)$ & $184(61)$ & $178(59)$ \\
\hline Generalised anxiety disorder & $232(74)$ & $231(73)$ & 209 (68) \\
\hline Panic syndrome (past month) & $123(43)$ & $146(50)$ & $132(46)$ \\
\hline Social phobia & $94(31)$ & $103(34)$ & $93(31)$ \\
\hline Post-traumatic stress disorder & $72(23)$ & $63(20)$ & $62(20)$ \\
\hline Panic syndrome (lifetime) & $54(17)$ & $61(20)$ & $56(18)$ \\
\hline \multicolumn{4}{|l|}{ Education, $n(\%)$} \\
\hline Primary school & $15(5)$ & $9(3)$ & $11(4)$ \\
\hline Technical school & $40(13)$ & $39(12)$ & $33(11)$ \\
\hline Secondary school (year 12) & $79(25)$ & $71(22)$ & $75(24)$ \\
\hline Post-secondary 2 years & $52(16)$ & $62(20)$ & $62(20)$ \\
\hline Tertiary & $129(41)$ & $135(43)$ & $128(41)$ \\
\hline \multicolumn{4}{|l|}{ Employment, $n$ (\%) } \\
\hline Employed & $222(70)$ & $225(71)$ & $239(77)$ \\
\hline Studying & $21(7)$ & $14(4)$ & $14(5)$ \\
\hline Unemployed & $39(12)$ & $44(14)$ & $36(11)$ \\
\hline Old age pension & $9(3)$ & $5(2)$ & $2(1)$ \\
\hline Disability pension & $4(1)$ & $12(4)$ & $6(2)$ \\
\hline $\begin{array}{l}\text { Current sick leave } \\
\text { ( } 1 \text { month or more) }\end{array}$ & $21(7)$ & $15(5)$ & $14(4)$ \\
\hline \multicolumn{4}{|l|}{ Health status, $n(\%)$} \\
\hline Daily activities affected & $142(45)$ & $120(38)$ & $113(36)$ \\
\hline Moderate or severe pain & $213(67)$ & $207(66)$ & $219(70)$ \\
\hline Highly worried & $121(38)$ & $127(40)$ & $117(37)$ \\
\hline \multicolumn{4}{|l|}{ Substance use } \\
\hline Hazardous drinking & $73(23)$ & $66(21)$ & $72(23)$ \\
\hline Regular binge drinking & $49(16)$ & $36(11)$ & $34(11)$ \\
\hline Daily tobacco use & $63(20)$ & $70(22)$ & $61(20)$ \\
\hline Antidepressant use, $n$ (\%) & $92(31)$ & $94(31)$ & $69(24)$ \\
\hline
\end{tabular}

\section{Depression severity}

Table 3 shows the group $\times$ time interaction effects on depression severity (standardised MADRS scores) at the interim and endpoint assessments. Depression severity reduced significantly in all three groups from baseline to 3 months and from baseline to 12 months (Fig. 2). The largest improvements were observed at the interim assessment, when the standardised depression severity scores had reduced more in the exercise group (Hedges' $g=0.57$, 95\% CI $0.41-0.73)$ and the ICBT group ( $g=0.66,95 \%$ CI $0.50-$ 0.82 ) compared with usual care. These group differences were maintained at the long-term follow-up assessment for both exercise (Hedges' $g=0.24,95 \%$ CI $0.08-0.40$ ) and ICBT (Hedges'

\begin{tabular}{|c|c|c|c|}
\hline \multirow[b]{2}{*}{ Assessment } & \multicolumn{3}{|c|}{ MADRS score: mean (s.d.) } \\
\hline & Physical exercise $(n=316)$ & ICBT $(n=317)$ & Treatment as usual $(n=312)$ \\
\hline Baseline & $22.2(6.9)$ & $21.9(7.0)$ & $20.8(7.2)$ \\
\hline Interim (3 months) & $11.3(7.9)$ & $11.2(7.3)$ & $13.9(8.9)$ \\
\hline End-point (12 months) & $10.8(7.6)$ & $9.8(7.8)$ & $11.1(8.7)$ \\
\hline
\end{tabular}




\begin{tabular}{|c|c|c|c|c|c|c|}
\hline & Beta (s.e.) & $95 \% \mathrm{Cl}$ & $P$ & OR & $95 \% \mathrm{Cl}$ & $P$ \\
\hline \multicolumn{7}{|l|}{ Primary end-point ${ }^{a}$} \\
\hline Physical exercise & $-0.34(0.10)$ & -0.55 to -0.13 & 0.001 & 1.44 & 0.78 to 2.69 & 0.25 \\
\hline ICBT & $-0.34(0.10)$ & -0.55 to -0.14 & 0.001 & 1.50 & 0.81 to 2.79 & 0.20 \\
\hline \multicolumn{7}{|l|}{ Interim assessment ${ }^{a}$} \\
\hline Physical exercise & $-0.60(0.11)$ & -0.82 to -0.39 & $<0.0001$ & 2.29 & 1.23 to 4.26 & 0.009 \\
\hline ICBT & $-0.52(0.11)$ & -0.73 to -0.31 & $<0.0001$ & 2.30 & 1.24 to 4.26 & 0.008 \\
\hline
\end{tabular}

$g=0.46,95 \%$ CI $0.31-0.62)$. The quadratic effect of time was significant in the regression model $(\beta=0.56, P<0.0001)$. A similar trend was found using the proportion of participants 1 s.d. or more below the baseline average (Table 3, Fig. 3). In this model, however, the treatment group differences were no longer significant at the follow-up assessment. A sensitivity analysis comparing the imputed data with a complete case analysis $(n=945)$ revealed trends comparable to the main analyses (see online Table DS1). The subgroup analysis comprising only patients with concurrent depression and anxiety $(n=633$, twothirds of the total sample) indicated a similar trend; however, the interaction effect of exercise at 12 months fell slightly below statistical significance $(\beta=-0.22,95 \%$ CI $-0.49-0.03$, $P=0.08$ ). The difference between exercise and ICBT at 12 months in the subgroup analysis was not significant (interaction term: $\beta=0.16$, 95\% CI $-0.08-0.42, P=0.2$; see online Table DS2). There was no difference in the proportion of participants reducing their initial MADRS score by $50 \%$ or more at 12 months: usual care $47 \%$ (s.e. $=0.032$ ), ICBT $47 \%$ (s.e. $=0.031$ ), exercise $50 \%$ (s.e. $=0.031)$.

\section{Discussion}

This is the first community-based RCT to compare the long-term effectiveness of exercise and ICBT with usual care for depression. All three interventions were associated with reductions in depression severity at 12-month follow-up. The largest treatment effect was obtained at the interim assessment ( 3 months), when severity had reduced significantly more in the exercise and ICBT

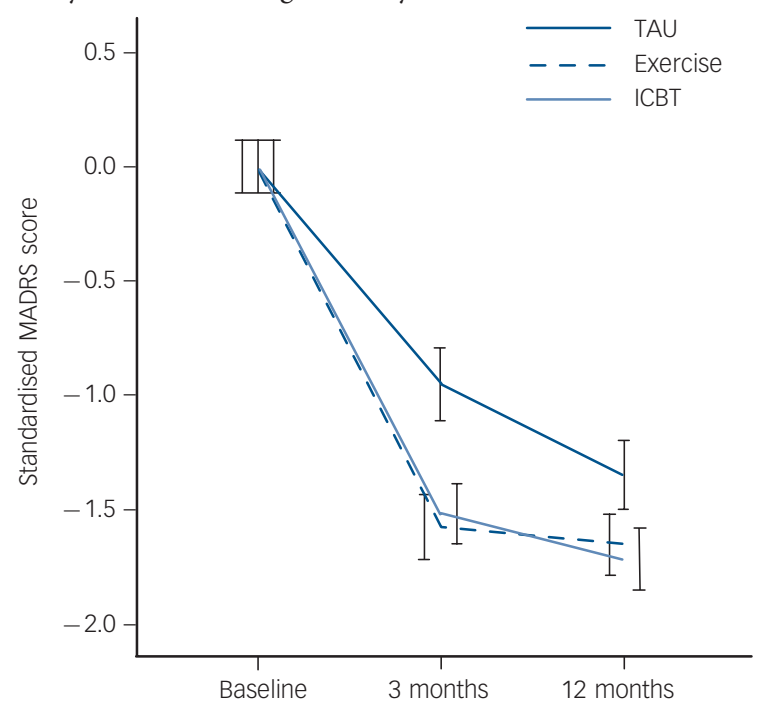

Fig. 2 Reduction in depression severity categorised by treatment group. ICBT, internet-based cognitive-behavioural therapy; MADRS, Montgomery-Åsberg Depression Rating Scale; TAU, treatment as usual. groups compared with usual care. Importantly, the long-term follow-up assessment reported in this study adds the observation that the short-term treatment benefits reported previously were maintained 9 months later. ${ }^{15}$ Taking into account the quadratic trend in these data, a conservative interpretation of the findings is that exercise and ICBT are at least equally effective long-term treatments for mild to moderate depression compared with usual care by a physician. The improvements were also clinically relevant. Depression scores reduced in the exercise and ICBT groups from a 'moderate' level at pre-treatment (scores 21.9-22.2) to a 'mild' level at post-treatment (11.2-11.3) and the study end-point $(9.8-10.8) .{ }^{17}$ A notable limitation of the study is that information about the number of patients who declined to participate and their characteristics is unknown.

\section{Comparison with other studies}

A promising finding is that exercise alone was associated with significantly larger long-term reductions in depression severity compared with usual care. 'Treatment as usual' in this trial was not a control group equivalent, but rather an active condition in which most patients regularly received face-to-face counselling. This suggests that the results are not uniquely attributable to greater support or monitoring within the exercise intervention. Some studies have not observed significant improvements in depression following exercise. For example, a recent community-based trial in the UK involving 361 adults aged 18-69 years found that the addition of a facilitated physical activity intervention to usual care did not improve depression outcomes. ${ }^{26}$ However, an important

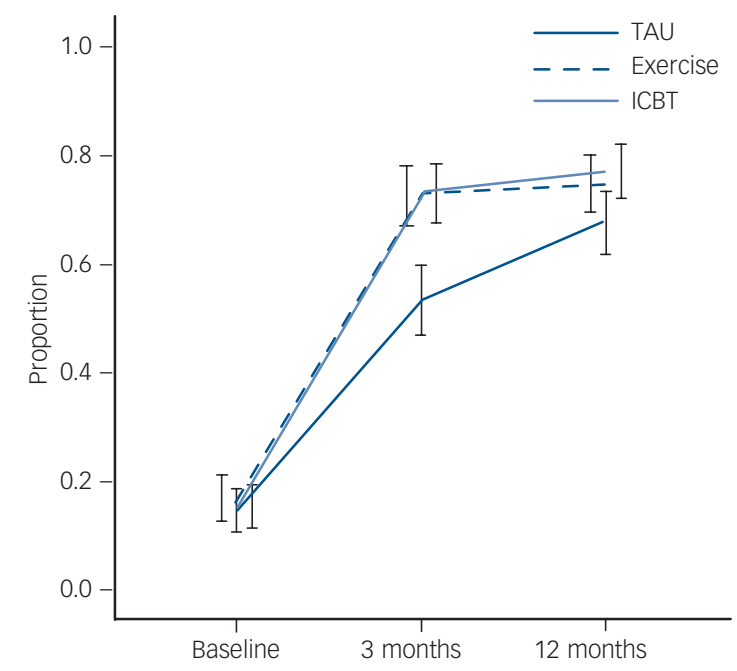

Fig. 3 Improvement in depression severity categorised by treatment group: proportion of participants with depression severity scores at least 1 s.d. lower than the baseline group average. ICBT, internet-based cognitive-behavioural therapy; TAU, treatment as usual. 
feature of our trial was the inclusion of structured, supervised exercise sessions for the duration of the study. This particular feature has been linked to better outcomes in previous exercise treatment studies. ${ }^{27}$

The exercise-depression research now spans several decades, and supports the conclusion that regular physical activity is associated with meaningful improvements in mental health. A recent Cochrane review of 35 RCTs $(n=1356)$ comparing exercise with no treatment or a control intervention reported a pooled standardised mean difference of $-0.62(95 \%$ CI -0.81 to -0.42 ), indicating a moderate clinical effect of exercise. ${ }^{3}$ However, a notable limitation of previous research is that most studies are based on small participant samples with short-term (post-treatment) follow-up assessments only. Thus, it has remained unclear whether prescribed exercise for depression has sustained treatment benefits equivalent to usual care. Our findings bridge this knowledge gap by demonstrating that, compared with usual care, exercise had reductions of similar magnitude at posttreatment assessment (Hedges' $g=0.57$ ) and smaller but statistically significant ongoing reductions at 12-month follow-up $(g=0.24)$. The benefits of exercise in this population extend beyond alleviating depressive symptoms. ${ }^{28}$ For instance, people with depression are at increased risk of diabetes and of premature mortality from cardiovascular disease, ${ }^{29,30}$ and in the general population exercise is comparably effective to pharmacological interventions in preventing cardiovascular disease and related premature mortality. ${ }^{31}$

Our findings also indicate that clinician-supported ICBT resulted in significant, small to moderate improvements in depression severity compared with usual care (at 3 months Hedges' $g=0.66$; at 12 months $g=0.46$ ). These findings add to an expanding literature supporting internet-based treatments for depression, ${ }^{12,32}$ and provide clinically relevant information about the long-term effectiveness of supported ICBT interventions. Strong associations have been reported between the degree of support provided in ICBT trials and better mental health outcomes. ${ }^{14}$ For example, Berger et al (2011) reported small to moderate effects favouring guided compared with unguided ICBT $^{33}$ A recent RCT also suggested that tailored ICBT may confer greater treatment benefits for patients with high comorbidity. ${ }^{13}$ In recognition of these potential benefits, the intervention reported here included individually tailored modules and limited clinician support.

Compared with exercise, however, and seen within the wider purview of treatment research, ICBT remains a relatively new phenomenon; some studies have failed to demonstrate benefits. A recent study conducted in primary care using a standardised 12-week clinician-supported ICBT programme for depression found no benefit compared with treatment as usual. ${ }^{34}$ Currently, ICBT is a treatment option supported primarily by efficacy studies; empirical support based on effectiveness trials conducted in primary care settings remains scarce. ${ }^{35,36}$ A notable limitation of previous ICBT research has been the absence of an active treatment comparison group in long-term effectiveness trials.

\section{Strengths and limitations}

Strengths of our investigation are the large participant sample, RCT design, comparison of three treatments, masked assessments and high questionnaire response rates. As noted, the inclusion of a 1-year follow-up assessment to measure sustained treatment effects is an advance over many previous RCTs. The randomisation was performed remotely and participants were clinically assessed in addition to self-ratings. The inclusion of patients using medication is both a strength and a weakness; it makes the intervention less 'pure' but increases the external validity of the findings. Clinicians facing treatment decisions are likely to encounter patients with diverse treatment backgrounds, including many who are already taking antidepressant medication.

Several limitations are also acknowledged. Most notable is that the number of invited participants who declined is unknown as it was not feasible to collect these data. One potential consequence is that the final participant sample may have consisted of individuals with characteristics favouring stronger outcomes in the exercise and ICBT groups, such as a positive attitude towards novel depression treatments. Although this possibility cannot be ruled out, the effect sizes shown here are comparable to those reported in similar studies. ${ }^{3}$ Consistent with previous work, we have focused on depressive symptoms; however, changes in functional outcomes associated with depression are also relevant. ${ }^{37}$ We have previously published interim effects of the interventions on work capacity. ${ }^{14}$ In the present study we compared exercise and ICBT with usual care for depression. As in previous trials, 'treatment as usual' was determined by individual clinicians, resulting in a degree of treatment heterogeneity (both type and frequency). Importantly, however, the inclusion of a usual care group enabled the comparative effectiveness of two alternative treatments for depression to be explored. Another study limitation is that despite ongoing monitoring and support, adherence to the exercise and ICBT interventions was suboptimal. A likely explanation for the lower adherence rate in the exercise group $(33 \%)$ compared with ICBT $(60 \%)$ is that some patients chose to exercise in their own time outside the scheduled exercise sessions. Finally, the analyses presented here do not allow any firm conclusions regarding the specific effects of the two interventions. The effects could be explained, to a greater or lesser extent, by non-specific factors such as contact with the internet therapist or the physical exercise trainer, or expectations raised by being randomised to one of the active treatments. As previously noted, however, the usual care group in this study was an active intervention involving ongoing contact with a physician, psychologist or counsellor. As a first step, we have reported the overall effect of the three interventions on depression severity.

\section{Clinical implications}

Compared with usual care by a physician, prescribed exercise and clinician-supported ICBT are at least equally effective long-term treatment alternatives for adults with mild to moderate depression. Despite strong evidence supporting the effectiveness of exercise to treat depression, and the availability of guidelines for exercise prescription, ${ }^{38,39}$ physical activity is rarely used to treat mental health problems. A recent Swedish study found that only 1 out of 1000 visits to primary healthcare resulted in an exercise prescription. ${ }^{40}$ Our results also support previous studies demonstrating positive effects of internet-based treatments for depression in adults. Additional community-based effectiveness trials of exercise and ICBT are recommended. Future studies should include functional outcomes such as work capacity and quality of life, and examine plausible biological and psychological mechanisms.

Mats Hallgren, PhD, Division of Epidemiology and Public Health Intervention Research (EPHIR), Department of Public Health Sciences, Karolinska Institute; Björg Helgadóttir, MSc, Department of Public Health Sciences, Karolinska Institute, Solna, Sweden; Matthew P. Herring, PhD, Department of Physical Education and Sport Sciences, and Health Research Institute, University of Limerick, Ireland; Zangin Zeebari, PhD, Department of Public Health Sciences, EPHIR, Karolinska Institute, Solna, sweden; Nils Lindefors, MD, PhD, Viktor Kaldo, PhD, Department of Clinica Solna, Sweden; Nils Lindefors, MD, PhD, Viktor Kaldo, PhD, Department of Clinica
Neuroscience, Karolinska Institute, Huddinge, Sweden; Agneta Öjehagen, PhD, Department of Clinical Sciences, Psychiatry, Lund University, Lund, Sweden; Yvonne Forsell, MD, PhD, Department of Public Health Sciences, EPHIR, Karolinska Institute, Solna, sweden

Correspondence: Mats Hallgren, Widerströmskahuset pl.10, Tomtebodavägen 18A, Karolinska Institutet, Solna 171 77, Sweden. Email: Mats.hallgren@ki.se

First received 26 Oct 2015, final revision 1 Apr 2016, accepted 21 May 2016 


\section{Funding}

The Regassa project is funded by Stockholm, Skåne, Kronoberg, Västra Götaland, Blekinge and Västmanland county councils, and REHSAM through the Vårdal Foundation. M.H. is supported by a postdoctoral grant from the Brain Foundation Sweden (Hjärnfonden). The funding sources had no role in study design, data collection, data analysis, data interpretation or writing of the report. The corresponding author had full access to all data in the study and had final responsibility for the decision to submit for publication.

\section{Acknowledgements}

The authors would like to thank staff in all six county councils for their tremendous efforts, especially Elisabeth Erwall. We also thank Dr Andreas Lundin for his comments on an earlier version of this paper.

\section{References}

1 Forsell Y. The pathway to meeting need for mental health services in Sweden. Psychiatr Serv 2006; 57: 114-9.

2 Sawada N, Uchida H, Suzuki T, Watanabe K, Kikuchi T, Handa T, et al. Persistence and compliance to antidepressant treatment in patients with depression: a chart review. BMC Psychiatry 2009; 9: 38.

3 Cooney GM, Dwan K, Greig CA, Lawlor DA, Rimer J, Waugh FR, et al. Exercise for depression. Cochrane Database Syst Rev 2013; 9: CD004366.

4 Herring MP, Puetz TW, O'Connor PJ, Dishman RK. Effect of exercise training on depressive symptoms among patients with a chronic illness: a systematic review and meta-analysis of randomized controlled trials. Arch Intern Med 2012; 172: 101-11.

5 Conn VS. Depressive symptom outcomes of physical activity interventions: meta-analysis findings. Ann Behav Med 2010; 39: 128-38.

6 Dunn AL, Jewell JS. The effect of exercise on mental health. Curr Sports Med Rep 2010; 9: 202-7.

7 Bjornebekk A, Mathe AA, Brene S. The antidepressant effect of running is associated with increased hippocampal cell proliferation. Int $J$ Neuropsychopharmacol 2005; 8: 357-68.

8 Eyre HA, Papps E, Baune BT. Treating depression and depression-like behavior with physical activity: an immune perspective. Front Psychiatry 2013; 4: 3.

9 Barbour KA, Edenfield TM, Blumenthal JA. Exercise as a treatment for depression and other psychiatric disorders: a review. J Cardiopulm Rehabil Prev 2007; 27: 359-67.

10 Cuijpers $P$, van Straten A, Andersson G. Internet-administered cognitive behavior therapy for health problems: a systematic review. J Behav Med 2008; 31: 169-77.

11 Cuijpers $P$, van Straten A, Andersson G, van Oppen P. Psychotherapy for depression in adults: a meta-analysis of comparative outcome studies. J Consult Clin Psychol 2008; 76: 909-22.

12 Andersson G, Cuijpers P, Carlbring P, Riper H, Hedman E. Guided Internetbased vs. face-to-face cognitive behavior therapy for psychiatric and somatic disorders: a systematic review and meta-analysis. World Psychiatry 2014; 13: 288-95

13 Johansson R, Sjoberg E, Sjogren M, Johnsson E, Carlbring P, Andersson T, et al. Tailored vs. standardized internet-based cognitive behavior therapy for depression and comorbid symptoms: a randomized controlled trial. PLOS ONE 2012; 7: e36905.

14 Johansson R, Andersson G. Internet-based psychological treatments for depression. Expert Rev Neurother 2012; 12: 861-9.

15 Hallgren M, Kraepelien M, Ojehagen A, Lindefors N, Zeebari Z, Kaldo V, et al. Physical exercise and internet-based cognitive-behavioural therapy in the treatment of depression: randomised controlled trial. Br J Psychiatry 2015; 207: 227-34.

16 Kroenke K, Spitzer RL, Williams JB. The PHQ-9: validity of a brief depression severity measure. J Gen Intern Med 2001; 16: 606-13.

17 Montgomery SA, Asberg M. A new depression scale designed to be sensitive to change. Br J Psychiatry 1979; 134: 382-9.

18 Snaith RP, Harrop FM, Newby DA, Teale C. Grade scores of the Montgomery Åsberg Depression and the Clinical Anxiety Scales. Br J Psychiatry 1986; 148 599-601.
19 Sheehan DV, Lecrubier $\mathrm{Y}$, Sheehan $\mathrm{KH}$, Amorim $\mathrm{P}$, Janavs J, Weiller $\mathrm{E}$, et al. The Mini-International Neuropsychiatric Interview (M.I.N.I.): the development and validation of a structured diagnostic psychiatric interview for DSM-IV and ICD-10. J Clin Psychiatry 1998; 59 (suppl 20): 22-33.

20 Rabin R, de Charro F. EQ-5D: a measure of health status from the EuroQol Group. Ann Med 2001; 33: 337-43.

21 Saunders JB, Aasland OG, Babor TF, de la Fuente JR, Grant M. Development of the Alcohol Use Disorders Identification Test (AUDIT): WHO Collaborative Project on Early Detection of Persons with Harmful Alcohol Consumption - II. Addiction 1993; 88: 791-804.

22 Bergman $\mathrm{H}$, Kallmen $\mathrm{H}$. Alcohol use among Swedes and a psychometric evaluation of the alcohol use disorders identification test. Alcohol Alcohol 2002; 37: 245-51.

23 Garber CE, Blissmer B, Deschenes MR, Franklin BA, Lamonte MJ, Lee IM, et al. American College of Sports Medicine position stand. Quantity and quality of exercise for developing and maintaining cardiorespiratory, musculoskeletal, and neuromotor fitness in apparently healthy adults: guidance for prescribing exercise. Med Sci Sports Exerc 2011; 43: 1334-59.

24 Pfaff JJ, Alfonso H, Newton RU, Sim M, Flicker L, Almeida OP. ACTIVEDEP: a randomised, controlled trial of a home-based exercise intervention to alleviate depression in middle-aged and older adults. Br J Sports Med 2014; 48: 226-32.

25 Hedges LV, Olkin I. Statistical Methods for Meta-analysis. Academic Press, 1985.

26 Chalder M, Wiles NJ, Campbell J, Hollinghurst SP, Haase AM, Taylor AH, et al. Facilitated physical activity as a treatment for depressed adults: randomised controlled trial. BMJ 2012; 344: e2758.

27 Stanton R, Reaburn P. Exercise and the treatment of depression: a review of the exercise program variables. J Sci Med Sport 2014; 17: 177-82.

28 Knapen J, Vancampfort D, Morien Y, Marchal Y. Exercise therapy improves both mental and physical health in patients with major depression. Disabil Rehabil 2015; 37: 1490-5.

29 Vancampfort $D$, Mitchell AJ, De Hert M, Sienaert P, Probst M, Buys R, et al. Type 2 diabetes in patients with major depressive disorder: a meta-analysis of prevalence estimates and predictors. Depress Anxiety 2015; 32: 763-73.

30 Holt RI, de Groot M, Lucki I, Hunter CM, Sartorius N, Golden SH. NIDDK international conference report on diabetes and depression: current understanding and future directions. Diabetes Care 2014; 37: 2067-77.

$31 \mathrm{Naci} H$, Ioannidis JP. Comparative effectiveness of exercise and drug interventions on mortality outcomes: metaepidemiological study. BMJ 2013; 347: f5577.

32 Strom M, Uckelstam CJ, Andersson G, Hassmen P, Umefjord G, Carlbring P, et al. Internet-delivered therapist-guided physical activity for mild to moderate depression: a randomized controlled trial. Peer J 2013; 1: e178.

33 Berger T, Hämmerli K, Gubser N, Andersson G, Caspar F. Internet-based treatment of depression: a randomized controlled trial comparing guided with unguided self-help. Cogn Behav Ther 2011; 40: 251-66.

34 Kivi M, Eriksson MC, Hange D, Petersson EL, Vernmark K, Johansson B, et al. Internet-based therapy for mild to moderate depression in Swedish primary care: short term results from the PRIM-NET randomized controlled trial. Cogn Behav Ther 2014; 43: 289-98.

35 Handbook of Non Drug Intervention (HANDI) Project Team. Internet-based cognitive behaviour therapy for depression and anxiety. Aust Fam Physician 2013; 42: 803-4.

36 Griffiths F, Lindenmeyer A, Powell J, Lowe P, Thorogood M. Why are health care interventions delivered over the internet? A systematic review of the published literature. J Med Internet Res 2006; 8: e10.

37 Blake $\mathrm{H}$. Physical activity and exercise in the treatment of depression. Front Psychiatry 2012; 3: 106.

38 Professional Associations for Physical Activity. Physical Activity in the Prevention and Treatment of Disease. Swedish National Institute of Public Health, 2010

39 Rethorst $\mathrm{CD}$, Trivedi $\mathrm{MH}$. Evidence-based recommendations for the prescription of exercise for major depressive disorder. J Psychiatr Pract 2013; 19: $204-12$.

40 Kallings LV. [Physical activity on prescription - an underutilized resource. Statistics on prescription shows large variations between counties.] Lakartidningen 2012; 109: 2348-50. 